\title{
Comparison of sustained-release and rapid-release $\beta$-alanine formulations on changes in skeletal muscle carnosine and histidine content and isometric performance following a muscle-damaging protocol
}

\author{
Alyssa N. Varanoske ${ }^{1}$. Jay R. Hoffman ${ }^{1}$. David D. Church ${ }^{1}$. Nicholas A. Coker ${ }^{1}$ Kayla M. Baker ${ }^{1}$. Sarah J. Dodd ${ }^{1}$. \\ Roger C. Harris ${ }^{2} \cdot$ Leonardo P. Oliveira $^{3} \cdot$ Virgil L. Dawson ${ }^{4} \cdot$ Ran Wang $^{1} \cdot$ David H. Fukuda $^{1}$. Jeffrey R. Stout ${ }^{1}$
}

Received: 14 April 2018 / Accepted: 27 June 2018 / Published online: 12 July 2018

(C) The Author(s) 2018

\begin{abstract}
$\beta$-alanine supplementation increases muscle carnosine content and improves anaerobic exercise performance by enhancing intracellular buffering capacity. $\beta$-alanine ingestion in its traditional rapid-release formulation (RR) is associated with the symptoms of paresthesia. A sustained-release formulation (SR) of $\beta$-alanine has been shown to circumvent paresthesia and extend the period of supply to muscle for carnosine synthesis. The purpose of this investigation was to compare 28 days of SR and RR formulations of $\beta$-alanine $\left(6 \mathrm{~g} \mathrm{day}^{-1}\right)$ on changes in carnosine content of the vastus lateralis and muscle fatigue. Thirty-nine recreationally active men and women were assigned to one of the three groups: SR, RR, or placebo (PLA). Participants supplementing with SR and RR formulations increased muscle carnosine content by $50.1 \%$ ( $\left.3.87 \mathrm{mmol} \mathrm{kg}^{-1} \mathrm{ww}\right)$ and $37.9 \%$ (2.62 mmol kg-1 $\mathrm{ww})$, respectively. The change in muscle carnosine content in participants consuming SR was significantly different $(p=0.010)$ from those consuming PLA, but no significant difference was noted between RR and PLA $(p=0.077)$. Although participants ingesting SR experienced a $16.4 \%$ greater increase in muscle carnosine than RR, fatigue during maximal voluntary isometric contractions was significantly attenuated in both SR and RR compared to PLA ( $p=0.002$ and 0.024 , respectively). Symptoms of paresthesia were significantly more frequent in RR compared to SR, the latter of which did not differ from PLA. Results of this study demonstrated that only participants consuming the SR formulation experienced a significant increase in muscle carnosine. Differences in the muscle carnosine response between these formulations may have practical significance for athletic populations in which small changes may have important implications on performance.
\end{abstract}

Keywords Supplementation $\cdot$ Histidine $\cdot$ Ergogenic Aid $\cdot \beta$-Alanine $\cdot$ Intracellular buffering capacity $\cdot$ Paresthesia

\section{Introduction}

Handling Editor: W. Derave.

Jay R. Hoffman

Jay.Hoffman@ucf.edu

1 Sport and Exercise Science, University of Central Florida, 12494 University Blvd, Orlando, FL 32816-1250, USA

2 Junipa Ltd, Newmarket, Suffolk, UK

3 Department of Orthopedics and Rehabilitation Medicine, University of Chicago Medicine and Biological Sciences, Chicago, IL, USA

4 Department of Internal Medicine, University of Central Florida College of Medicine, Orlando, FL, USA
Carnosine ( $\beta$-alanyl-L-histidine) is an intramuscular dipeptide consisting of $\beta$-alanine and L-histidine (Dunnett and Harris 1999). Of these, $\beta$-alanine appears to be rate limiting to carnosine formation (Harris et al. 2006). Although histidine is an essential amino acid in humans, it is found in sufficient supply in the body, whereas $\beta$-alanine is not (Dunnett and Harris 1999; Harris et al. 2006). Endogenous $\beta$-alanine production occurs principally through hepatic degradation of uracil and results in only small amounts of carnosine synthesis in muscle (Baguet et al. 2010). $\beta$-alanine is also present in meat and fish products; however, relying on $\beta$-alanine intake from one's diet may not be sufficient or efficient, as $200 \mathrm{~g}$ of chicken would need to be consumed to increase 
the plasma bioavailability of $\beta$-alanine concentration to the same extent as an $800 \mathrm{mg}$ supplement (Harris et al. 2006). Therefore, exogenous supplementation with $\beta$-alanine seems to be the most effective at increasing muscle carnosine concentrations (Harris et al. 2006; Dunnett and Harris 1999).

$\beta$-alanine has been widely explored as a nutritional supplement for improving exercise performance in various populations. Research examining the ergogenic effects of $\beta$-alanine supplementation has been completed in athletes (Baguet et al. 2010; de Salles Painelli et al. 2013; Hoffman et al. 2008; Derave et al. 2007), recreationally active individuals (Hill et al. 2007; Smith-Ryan et al. 2012; Stout et al. 2007), older adults (McCormack et al. 2013; Stout et al. 2008) and in military personnel (Hoffman et al. 2014; $2015 \mathrm{a}$ ), showing that $\beta$-alanine supplementation is most effective at improving high-intensity exercise performance lasting between 0.5 and $10 \mathrm{~min}$ in duration (Saunders et al. 2017). The increase in anaerobic performance with $\beta$-alanine supplementation is a direct result of the increase in intramuscular carnosine concentrations rather than $\beta$-alanine itself (Hoffman et al. 2015b). Carnosine acts as a buffer of $\mathrm{H}^{+}$, decreasing exercise-induced acidosis and, therefore, prolonging high-intensity exercise duration (Abe 2000). Additionally, recent investigations by Hannah et al. (2015) and Jones et al. (2017) have suggested that $\beta$-alanine supplementation and subsequent increases in muscle carnosine content can result in a reduction in muscle relaxation time, which may be especially beneficial for repeated bouts of exercise. This can potentially augment force production and provide a further mechanism for the improvements in exercise performance with $\beta$-alanine supplementation.

Investigations examining $\beta$-alanine supplementation have generally used daily dosing regimens ranging between 1.6 and $6.4 \mathrm{~g} \mathrm{day}^{-1}$ (Hoffman et al. 2018). Higher doses of the rapid release formulation have not been examined due to the greater risk of symptoms of paresthesia (Hoffman et al. 2018), which include flushing, irritation, and pricking of the skin. At times, this has resulted in an ingestion pattern requiring small doses to be consumed multiple times per day (Hill et al. 2007). Recently, a sustained-release formulation of $\beta$-alanine has become available. The advantage of a sustained-release formulation is that it can reduce symptoms of paresthesia and potentially allow for a greater daily dose to be consumed. Decombaz and et al. (2012) compared $1.6 \mathrm{~g}$ of rapid-release and sustained release formulations of $\beta$-alanine and reported no difference in the area under the curve for plasma $\beta$-alanine concentrations for $6 \mathrm{~h}$ following ingestion. In addition, the sustained-release $\beta$-alanine formulation resulted in a delay in the peak concentration of $\beta$-alanine in plasma and a longer retention time compared to the rapid-release formulation.

It has been suggested that circulating $\beta$-alanine may bind to Mas-related G-protein (Mrg) coupled receptors within the body, which have a high affinity for itch-inducing ligands (Bader et al. 2014). $\beta$-alanine is thought to bind specifically, to $\mathrm{MrgD}$, a receptor that is primarily expressed in the sensory dorsal root ganglion neurons that is found under the skin (Crozier et al. 2007). The MrgD receptor is extensively involved in pruriception and may be responsible for the itchy sensation that is associated with $\beta$-alanine supplementation (Bader et al. 2014; Crozier et al. 2007). Symptoms of paresthesia may indicate that some of the ingested $\beta$-alanine is bound to $\mathrm{MrgD}$ receptors; however whether this affects $\beta$-alanine availability to the muscle is unknown. In comparison to a sustained-release formulation of $\beta$-alanine which extends the $\beta$-alanine concentrations in the plasma for longer duration, a rapid-release formulation is likely to have a higher proportion of its content excreted via the urine (Decombaz et al. 2012), or have another unknown metabolic fate (Stegen et al. 2013). The sustained-release formulation may, therefore, provide greater $\beta$-alanine availability to the muscle and result in a greater percentage from each single dose of supplemented $\beta$-alanine to be retained in muscle as carnosine. However, $\beta$-alanine retention may not be a good indication of carnosine formation. In a previous study, comparing the effects of sustained-release $\beta$-alanine supplementation in a tablet to rapid-release $\beta$-alanine supplementation in a powder filled gel capsule ( $3.2 \mathrm{~g} \mathrm{day}^{-1}$ for 46 days) reported similar increases in carnosine content with both formulations (Stegen et al. 2013). Despite similar increases in carnosine content, the effects of each formulation on exercise performance and muscle metabolites were not examined in this investigation. Furthermore, differences in supplement formulation (tablet vs. gel capsule) may also have affected absorption rates.

Recent research by Blancquaert et al. (2017) suggested that intramuscular histidine availability is limited. These researchers observed that 23 days of $\beta$-alanine supplementation $\left(6.0 \mathrm{~g} \mathrm{day}^{-1}\right)$ resulted in a decline in muscle histidine (Blancquaert et al. 2017). However, recent investigations by Church et al. (2017) and Varanoske et al. (2017a) reported contrasting results, as 4 -weeks of $\beta$-alanine $\left(6.0 \mathrm{~g} \mathrm{day}^{-1}\right)$ supplementation in recreationally active individuals resulted in no decrease in muscle histidine. A decrease in muscle histidine levels may compromise physiological function by reducing protein synthesis and lowering hematocrit and hemoglobin levels (Kriengsinyos et al. 2002). An interesting difference between these studies is that Church et al. (2017) and Varanoske et al. (2017a) utilized sustained-release formulations of $\beta$-alanine, whereas Blancquaert et al. (2017) used rapid-release $\beta$-alanine. It is possible that the different pharmacokinetic properties of sustained-release and rapidrelease $\beta$-alanine may affect concentrations of intramuscular histidine content in a different manner. Therefore, the purpose of this study was to compare 4-weeks of sustainedand rapid-release formulations of $\beta$-alanine supplementation 
$\left(6 \mathrm{~g} \mathrm{day}^{-1}\right)$ on changes in muscle carnosine, $\beta$-alanine, and histidine in young adults. In addition, the effect of these different $\beta$-alanine formulations on changes in muscle performance was also examined.

\section{Methods}

\section{Experimental design}

Each participant reported to the Human Performance Laboratory at the University of Central Florida on two occasions separated by a period of four weeks ( 28 days). Informed consent was obtained from all individual participants included in the study. On the first day of testing, each participant was randomly assigned into one of three groups: rapid-release $\beta$-alanine (RR), sustained-release $\beta$-alanine (SR), or placebo (PLA). This was a double-blinded experimental design. Each participant was instructed to consume $6 \mathrm{~g}$ day $^{-1}$ of their respective supplement for a total ingestion of $168 \mathrm{~g}$. Prior to each testing session, participants were instructed to fast for a minimum of $2 \mathrm{~h}$ and avoid lower body physical activity for $48 \mathrm{~h}$ prior to testing. During each visit, body composition measures and a muscle biopsy were obtained from participants prior to completing a fatiguing knee extensor protocol.

\section{Participants}

Thirty-seven physically active men and women were recruited for this study. Participants were stratified into one of the three groups (SR, RR, or PLA) in a counterbalanced fashion based on the peak torque (PT) values obtained during unilateral maximal voluntary isometric contraction (MVIC) performed on the first testing day. Participants were instructed to maintain normal food and exercise habits throughout the duration of the study. This investigation was approved by the New England Institutional Review Board for human subjects, and all procedures were in accordance with the ethical standards of the 1964 Helsinki Declaration and its later amendments. Following an explanation of all procedures, risks, and benefits, each participant provided their written informed consent to participate in the study. All participants were required to be free of any physical limitations (as determined by medical history questionnaire and PAR-Q) and not to have consumed $\beta$-alanine for at least 9 weeks prior to enrollment in the study. Although being a vegetarian was not part of the exclusion criteria, none of the participants recruited reported to be a vegetarian. The demographics of each group can be seen in Table 1 .

\section{Supplementation protocol}

Participants in each group were provided with $2 \mathrm{~g}$ of either RR $\beta$-alanine, SR $\beta$-alanine or PLA three times per day (total $\beta$-alanine intake was $6 \mathrm{~g} \mathrm{day}^{-1}$ ). Participants were instructed to consume their supplements with regular meals (breakfast, lunch, dinner). Both RR and SR supplements were provided in tablet form $(1 \mathrm{~g})$ and were identical in appearance. Participants in the placebo group were provided with the same number of tablets that were also identical in appearance to the $\beta$-alanine tablets. Active and placebo tablets were provided by Natural Alternatives International (Carlsbad, CA, USA). Supplementation compliance was tracked by selfreported supplement logs and by inspection of the number of tablets remaining in each bottle upon return for follow-up testing. If participants fell below $90 \%$ compliance by either method, they were removed from the final analysis.

\section{Anthropometric measurements}

Anthropometric measurements were assessed for each participant during both visits to the laboratory. Upon arrival to the laboratory, participants were instructed to void their bladder in order to properly assess body composition. Height $( \pm 0.1 \mathrm{~cm})$ and body mass $( \pm 0.1 \mathrm{~kg})$ were determined using
Table 1 Characteristics of participants included in the final data analysis

\begin{tabular}{llrllll}
\hline Group & Subjects & $N$ & Age (years) & Height $(\mathrm{m})$ & Body mass $(\mathrm{kg})$ & Body fat $(\%)$ \\
\hline SR & All & 12 & $22.8 \pm 2.5$ & $1.70 \pm 0.09$ & $70.3 \pm 10.2$ & $22.3 \pm 7.2$ \\
& Males & 6 & $21.8 \pm 1.2$ & $1.78 \pm 0.06$ & $79.1 \pm 5.0$ & $19.3 \pm 9.2$ \\
& Females & 6 & $23.7 \pm 3.3$ & $1.62 \pm 0.05$ & $61.6 \pm 4.2$ & $25.4 \pm 2.7$ \\
RR & All & 9 & $21.9 \pm 2.4$ & $1.71 \pm 0.09$ & $74.0 \pm 15.1$ & $21.0 \pm 8.4$ \\
& Males & 5 & $22.2 \pm 3.3$ & $1.75 \pm 0.07$ & $82.7 \pm 14.3$ & $15.0 \pm 5.2$ \\
& Females & 4 & $21.5 \pm 1.0$ & $1.67 \pm 0.09$ & $63.2 \pm 7.1$ & $28.6 \pm 4.0$ \\
PLA & All & 8 & $23.4 \pm 3.0$ & $1.72 \pm 0.10$ & $76.1 \pm 15.3$ & $24.3 \pm 9.9$ \\
& Males & 4 & $23.5 \pm 2.6$ & $1.78 \pm 0.11$ & $84.9 \pm 16.5$ & $17.0 \pm 5.5$ \\
& Females & 4 & $23.3 \pm 3.8$ & $1.67 \pm 0.05$ & $67.2 \pm 8.1$ & $31.7 \pm 7.2$ \\
Total & & 29 & $22.6 \pm 2.6$ & $1.71 \pm 0.09$ & $73.1 \pm 13.0$ & $22.5 \pm 8.2$ \\
\hline
\end{tabular}

$S R$ Sustained-release $\beta$-alanine, $R R$ rapid-release $\beta$-alanine, $P L A$ placebo 
a Health-O-Meter Professional scale (Model 500 KL, Pelstar, Alsip, IL, USA). Body composition was assessed via air displacement plethysmography (BodPod ${ }^{\circledR}$, COSMED, Chicago, IL, USA). Anthropometric measures of each group are depicted in Table 1.

\section{Muscle biopsies}

The muscle biopsy procedures used in this investigation have been previously reported (Varanoske et al. 2017a, b; Church et al. 2017). Prior to testing, all participants were instructed to wear shorts on testing day to expose the upper portion of their thigh. Fine-needle muscle biopsies were obtained from the vastus lateralis (VL) muscle of the left leg at $50 \%$ of the straight-line distance between the lateral border of the patella and the greater trochanter of the femur (Townsend et al. 2016). A B-mode, linear probe ultrasound (General Electric LOGIQe, Wauwatosa, WI, USA), coated with transmission gel (Aquasonic ${ }^{\circledR} 100$, Parker Laboratories, Inc., Fairfield, NJ, USA) was used to determine muscle thickness and subcutaneous adipose tissue thickness at the aforementioned location on the leg to provide guidance prior to biopsy procedures. The biopsy area was washed with antiseptic soap and cleaned with rubbing alcohol. A small area of the clean skin approximately $2 \mathrm{~cm}$ in diameter was then anesthetized with a $2.0 \mathrm{~mL}$ subcutaneous injection of Lidocaine. The biopsy site was further cleansed by swabbing the area with betadine. Once anesthetized, a spring loaded reusable microbiopsy instrument with a disposable 14-gauge needle (Argon Medical Devices Inc., Plano, TX, USA) was inserted into the skin at an approximate depth of 1-2 cm to extract the muscle sample. Approximately, 5-6 muscle samples were extracted from each participant on each occasion, with the goal of obtaining about 15-20 mg of total wet tissue weight. After removal, each muscle sample was transferred to a petri dish placed on ice to trim adipose tissue from the muscle specimens. The remaining muscle specimens were then immediately frozen in liquid nitrogen and stored at $-80{ }^{\circ} \mathrm{C}$ for later analysis.

\section{Isometric testing and isokinetic muscle-fatiguing protocol}

The isometric testing and the muscle-fatiguing protocol used in this investigation have been previously reported (Varanoske et al. 2017a, b; Church et al. 2017). Due to the participants not being specifically trained in resistance exercise, an isokinetic dynamometer was used to elicit muscle fatigue and evaluate muscle strength while preventing injury. Briefly, as participants were reporting to the lab on a 2-h minimum fast, $8 \mathrm{oz}$ of a carbohydrate-containing beverage (60 calories, $16 \mathrm{~g}$ carbohydrates, $0 \mathrm{~g}$ fat, and $0 \mathrm{~g}$ protein) was provided to the participants following the muscle biopsy, 30-minutes prior to the isokinetic muscle-fatiguing protocol. Unilateral MVICs and an isokinetic muscle-fatiguing protocol were performed on an isokinetic dynamometer (System 4, Biodex Medical System, Inc., New York, NY, USA). To avoid any residual effects of the muscle biopsy, the right leg of each participant was tested. The lower portion of the leg was secured to the dynamometer arm just above the medial and lateral malleoli. Participants were seated in the dynamometer with a hip angle of $110^{\circ}$ and strapped to the chair at the waist, shoulders, and across the left thigh. Chair and dynamometer settings were adjusted for each participant but kept consistent between visits to properly align the axis of rotation of the knee with the lateral condyle of the femur. Range of motion was assessed for each participant. All participants were able to achieve a range of motion of $90-170^{\circ}$ without discomfort. The gravity effect of moment was measured at $120^{\circ}$ of knee flexion $\left(180^{\circ}\right.$ representing full extension) and subsequently corrected during testing (Beyer et al. 2016).

The isokinetic muscle-fatiguing protocol consisted of $5 \times 50$ maximal voluntary isokinetic unilateral knee extensions at a constant angular velocity of $180^{\circ} \mathrm{s}^{-1}$. Each contraction was initiated from a position of $90^{\circ}$ knee flexion and was continued to the point of full knee extension. After each extension, the lower leg was passively returned to the start position at $90^{\circ} \mathrm{s}^{-1}(\sim 1.5 \mathrm{~s}$ for a full cycle). Each bout of 50 contractions was separated by a 60 -s recovery period. Participants were encouraged during the first three contractions to make sure that they were contracting maximally from the start of each bout.

Two MVICs were performed prior to the isokinetic muscle-fatiguing protocol, separated by a period of $3 \mathrm{~min}$. Of the two MVICs performed prior to the isokinetic musclefatiguing protocol, the one that produced the greatest PT was saved and used for later analysis (MVIC1). Additionally, one MVIC was performed 10-s after the final set of the musclefatiguing protocol was completed (MVIC2). During these tests, the knee angle was fixed at $110^{\circ}$, and all MVICs were held for a total of $6 \mathrm{~s}$. PT was recorded during each MVIC. Differences in PT between MVIC1 and MVIC2 $(\Delta \mathrm{PT})$ were assessed at each time point to evaluate the effects of muscle fatigue.

Torque signals were sampled at $1 \mathrm{kHz}$ with a Biopac data acquisition system (MP150 Biopac Systems, Inc., Santa Barbara, CA, USA), recorded on a personal computer, and processed offline with custom written software (MATLAB, The MathWorks, Inc., Natick, MA, USA). PT was identified as the greatest torque achieved on the torque-time curve.

\section{Nutrient intake and dietary analysis}

Participants were instructed to maintain their normal dietary intake habits throughout the investigation. Total energy, 
macronutrient, and histidine intakes were monitored using recorded food logs during the 72-h period prior to each visit. The FoodWorks Dietary Analysis Software, Version 13 (The Nutrition Company, Long Valley, NJ, USA) was used to analyze dietary recalls.

\section{Side effects}

Participants were required to record side effects that were associated with consuming the supplement on a daily calendar. Side effects were recorded subjectively, through explanation of the symptom and by noting the time during which the symptom occurred. Side effects were analyzed by quantifying the total amount of days during which paresthesia was encountered over the 28-day supplementation period for each subject.

\section{Preparation of skeletal muscle tissue for high performance liquid chromatography (HPLC) analysis}

Muscle biopsy samples were homogenized with three volumes of $0.01 \mathrm{~N}$ hydrochloric acid (Sigma-Aldrich, St. Louis, MO, USA) and subsequently centrifuged at $4{ }^{\circ} \mathrm{C}$ for $20 \mathrm{~min}$ at $10,000 \mathrm{rpm}$. Muscle homogenates were deproteinized with three volumes of acetonitrile (BDH VWR Analytical, Radnor, PA, USA), and left to stand at $4{ }^{\circ} \mathrm{C}$ for $20 \mathrm{~min}$. Then, the sample was centrifuged at $4{ }^{\circ} \mathrm{C}$ for $10 \mathrm{~min}$ at $10,000 \mathrm{rpm}$. The supernatant was collected and subsequently analyzed.

\section{Determination of skeletal muscle carnosine}

The experimental methods were performed as described by Mora et al. (2007). Calibration standards were prepared in the range of $0.1-5 \mathrm{mM}$ by dilution of a stock $10 \mathrm{mM}$ solution. Chromatography was performed on an Agilent Infinity 1260 HPLC (Agilent Technologies, Santa Clara, CA, USA) and separation was carried out using an Atlantis hydrophilic interaction chromatography (HILIC) silica column $(4.6 \times 150 \mathrm{~mm}, 3 \mu \mathrm{m})$ from Waters (Milford, MA, USA) at room temperature. Mobile phase consisted of solvent A, containing $0.65 \mathrm{mM}$ ammonium acetate (Sigma-Aldrich, St. Louis, MO, USA), pH 5.5, in water/acetonitrile (25:75), and solvent $\mathrm{B}$, containing $4.55 \mathrm{mM}$ ammonium acetate, $\mathrm{pH} 5.5$, in water/acetonitrile (70:30). Solvents were filtered through a $0.22 \mu \mathrm{m}$ membrane filter and degassed prior to the analytical run. The separation conditions were a linear gradient from 0 to $100 \%$ of solvent B in 13 min at a flow rate of $1.4 \mathrm{~mL} \mathrm{~min}{ }^{-1}$. The column was equilibrated for $10 \mathrm{~min}$ under initial conditions before each injection. The separation was monitored using a diode array detector at a wavelength of $214 \mathrm{~nm}$ for carnosine and histidine. Peak areas were correlated to compound concentration by interpolation in the corresponding calibration curve. Duplication of retention times for a known standard was used to verify column equilibrium prior to analysis. Each sample was run in duplicate; the average intra-assay $\mathrm{CV}$ of carnosine was $1.62 \%$ and the inter-assay $\mathrm{CV}$ was $5.45 \%$.

\section{Determination of skeletal muscle histidine and $\beta$-alanine}

Calibration standards were prepared in the range of $1-0.01 \mathrm{mM}$ for histidine and $\beta$-alanine (Sigma-Aldrich, St. Louis, MO, USA). Norvaline (Nva; Ark Pharm, Arlington Heights, IL, USA) was used as an internal standard at a concentration of $0.1 \mathrm{mM} ; 5 \mu \mathrm{L}$ of Nva was added to $20 \mu \mathrm{L}$ of muscle homogenate prior to deproteinization. Afterwards, the supernatant was transferred to vials and automatic precolumn derivatization with ortho-phthalaldehyde (OPA; Agilent Technologies, Santa Clara, CA, USA) was performed at room temperature. Separation was carried out using a Poroshell $120 \mathrm{HPH}-\mathrm{C} 18$ column $(3.0 \times 100 \mathrm{~mm}$, $4 \mu \mathrm{m}$, Agilent Technologies, Santa Clara, CA, USA) at $40 \pm 0.8^{\circ} \mathrm{C}$. Mobile phase consisted of solvent A which contained $10 \mathrm{mM}$ sodium phosphate dibasic (Sigma-Aldrich, St. Louis, MO, USA), $10 \mathrm{mM}$ sodium tetraborate decahydrate (Alfa Aesar, Tewksbury, MA, USA), and $5 \mathrm{mM}$ sodium azide (BDH VWR Analytical, Radnor, PA, USA), $\mathrm{pH} 8.2$, in water, and solvent $B$ which contained acetonitrile, methanol (BDH VWR Analytical, Radnor, PA, USA), and water (45:45:10, v:v:v). Primary amino acids derivatized with OPA were detected at $338 \mathrm{~nm}$. Each sample was run in duplicate; the average intra-assay $\mathrm{CV}$ for $\beta$-alanine was $1.60 \%$, while the average intra-assay $\mathrm{CV}$ for histidine was $1.52 \%$.

\section{Statistical analysis}

Prior to statistical procedures, all data were assessed for normality, homogeneity of variance, and sphericity. If the assumption of sphericity was violated, a Greenhouse-Geisser correction was applied. Analyses of covariance (ANCOVA) were used to analyze changes in the dependent variables (nutrient intake, muscle carnosine, muscle histidine, muscle $\beta$-alanine, and $\Delta \mathrm{PT}$ ) based on the recommendations of Huck and McLean (1975) and Vincent and Weir (2012). PRE- and POST-values were used as the covariate and dependent variable, respectively. To analyze differences in supplement compliance, side effects, and participant characteristics between groups, a one-way ANOVA was performed. In the event of a significant $F$ ratio for any of these analyses, LSD post hoc comparisons were performed. Outliers were identified when values exceeded 1.5 times the interquartile range (Barbato et al. 2011). For all analyses, a criterion alpha level of $\alpha \leq 0.05$ was used to determine statistical significance and statistical software (SPSS 
V.21.0, Chicago, IL, USA) was used. Effect sizes for post hoc comparisons were calculated using statistical software (G*Power V.3.1.9.2) and were interpreted according to the recommendations of Cohen (1969). All data are reported as mean \pm standard deviation.

\section{Results}

\section{Participants}

Recruitment of participants, screening, and progression through the study are presented in Fig. 1. A total of 10 participants withdrew from the study prior to group assignment due to reasons unrelated to the investigation. One participant was removed from the final data analysis due to the lack of compliance to the supplementation protocol. Four participants were removed from the final data analysis because they were deemed to be outliers. Two additional participants from PLA were excluded from the final analysis due to inabilities to fulfill the time commitments of the study. Additionally, 3 subjects in RR were removed from the final analysis due to errors in data collection. Therefore, a total of 29 participants (SR $n=12$, RR $n=9$, PLA $n=8$ ) were included in the final analysis. The demographics of participants included in the final analysis are reported in Table 1 . No significant differences in any of the participant characteristics (age, height, body mass, body fat percentage) were observed $(p>0.05)$.

\section{Nutrient intake and dietary analysis}

Dietary analysis revealed that at PRE, the adjusted average nutrient intake for participants during the $72 \mathrm{~h}$ prior to the testing session was: $5137.5 \pm 1884.3 \mathrm{kcal}, 519.3 \pm 209.1 \mathrm{~g}$ of carbohydrate, $216.0 \pm 112.1 \mathrm{~g}$ of fat, $286.7 \pm 113.9 \mathrm{~g}$ of protein, and $2.01 \pm 1.45 \mathrm{~g}$ of histidine. No significant differences between the groups were shown at POST between any of the nutrient intake variables (kcal $F=0.168, p=0.847$, partial $\eta^{2}=0.013$; carbohydrate $F=0.347, p=0.710$, partial $\eta^{2}=0.027$; fat $F=0.513, p=0.605$, partial $\eta^{2}=0.039$; protein $F=0.079, p=0.924$, partial $\eta^{2}=0.006$; histidine $\left(F=1.129, p=0.339\right.$, partial $\left.\eta^{2}=0.083\right)$.

\section{Compliance and side effects}

No significant difference in supplement compliance was noted between the groups. The only reported side effect associated with consuming the supplement was symptoms of "tingling" or "itching", feelings generally associated with paresthesia. A statistically significant difference was

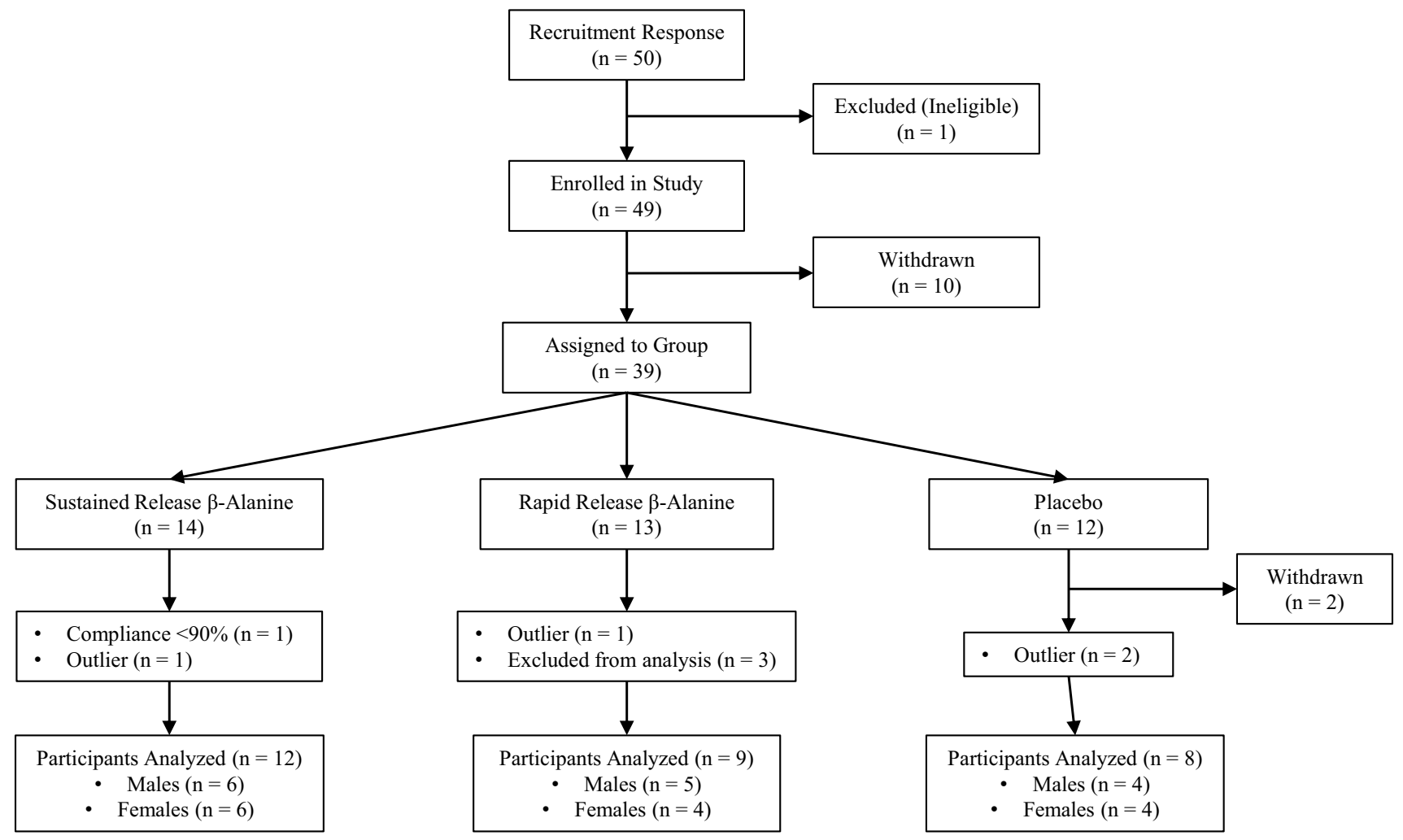

Fig. 1 Participant recruitment, sampling, and progression through the study. Supplementation compliance was prospectively set at $>90 \%$ throughout the duration of the study 
shown between the groups regarding the amount of paresthesia experienced over the course of the supplementation period $\left(F=46.703, p<0.001\right.$, partial $\left.\eta^{2}=0.781\right)$. Participants consuming RR formulation reported paresthesia on significantly more days ( $25.4 \pm 4.8$ days) compared to individuals consuming the SR formulation $(3.4 \pm 8.4$ days; $p<0.001, d=3.22)$ or those consuming PLA $(0.1 \pm 0.4$ days; $p<0.001, d=7.40)$. No significant differences in feelings of paresthesia were discovered between participants in SR and PLA $(p=0.245, d=0.56)$.

\section{Skeletal muscle carnosine content}

Changes in skeletal muscle carnosine are depicted in Fig. 2. At PRE, the average-adjusted muscle carnosine content was $7.63 \pm 2.62 \mathrm{mmol} \mathrm{kg}^{-1}$ ww. Significant differences $\left(F=3.911, p=0.033\right.$, partial $\left.\eta^{2}=0.238\right)$ in muscle carnosine were shown between groups at POST. Muscle carnosine content in participants consuming SR $\left(11.51 \pm 3.58 \mathrm{mmol} \mathrm{kg}^{-1} \mathrm{ww}\right)$ was significantly greater ( $p=0.010, d=1.26$ ) compared to participants consuming PLA $\left(6.96 \pm 3.61 \mathrm{mmol} \mathrm{kg}^{-1} \mathrm{ww}\right)$. No significant differences ( $p=0.077, d=0.90)$ were shown between participants consuming RR $\left(10.22 \pm 3.62 \mathrm{mmol} \mathrm{kg}^{-1} \mathrm{ww}\right)$ and those consuming PLA; however, increases in muscle carnosine for SR appeared to have a larger effect. Additionally, no statistically significant difference $(p=0.429, d=0.36)$ was noted for muscle carnosine values at POST between participants consuming the SR and RR formulations. The unadjusted change in muscle carnosine values from PRE to POST for participants consuming the SR formulation

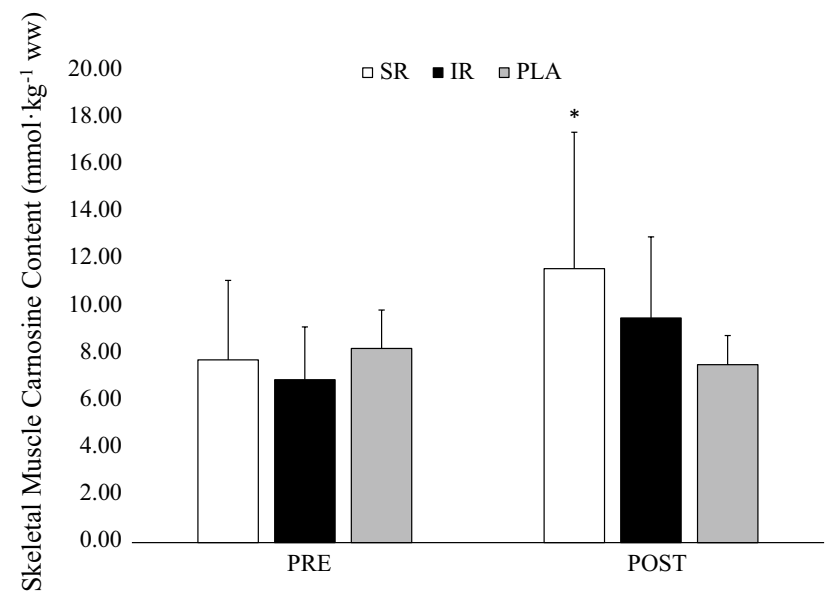

Fig. 2 Unadjusted values for PRE- and POST-supplementation skeletal muscle carnosine content. *Significantly greater than placebo after adjusting for the PRE covariate (adjusted PRE mean $=7.63 \mathrm{mmol} \mathrm{kg} \mathrm{kg}^{-1} \mathrm{ww}$ ). Results are reported as mean $\pm \mathrm{SD}$. $P R E$ before 28 days of supplementation; POST after 28 days of supplementation, $S R$ sustained-release formulation of $\beta$-alanine, $R R$ rapid-release formulation of $\beta$-alanine, $P L A$ placebo was $3.87 \pm 4.12 \mathrm{mmol} \mathrm{kg}^{-1} \mathrm{wW}$ and $2.62 \pm 3.91 \mathrm{mmol} \mathrm{kg}^{-1}$ ww in participants consuming the RR formulation. These changes reflected a 50.1 and $37.9 \%$ increase in carnosine content, respectively, corresponding to a $16.4 \%$ difference in carnosine content between dosing strategies from PRE values.

\section{Skeletal muscle histidine and $\beta$-alanine content}

Changes in skeletal muscle histidine and $\beta$-alanine are depicted in Figs. 3 and 4 , respectively. At PRE, the average-adjusted muscle histidine content was $0.34 \pm 0.17 \mathrm{mmol} \mathrm{\textrm {kg } ^ { - 1 }}$ ww. No significant differences $(F=0.082, p=0.921$,

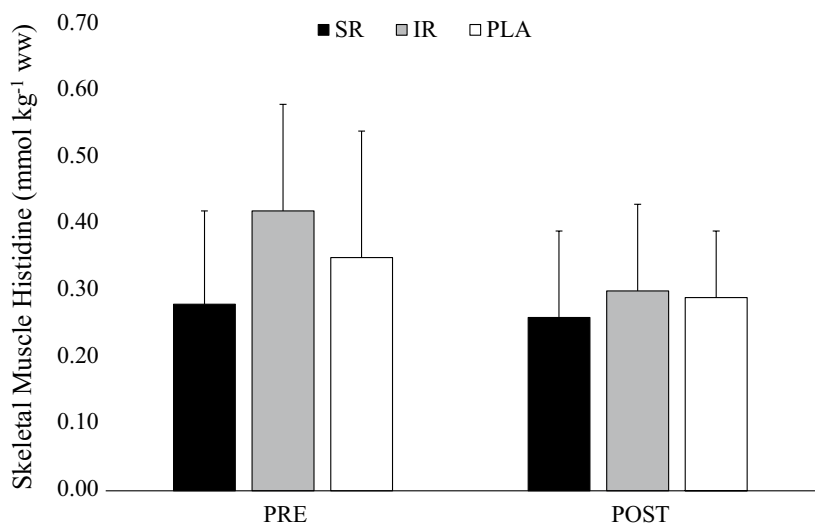

Fig. 3 Unadjusted values for PRE- and POST-supplementation skeletal muscle histidine content (adjusted PRE mean $=0.34 \mathrm{mmol} \mathrm{kg}^{-1}$ ww). Results are reported as mean \pm SD. PRE before 28 days of supplementation, $P O S T$ after 28 days of supplementation, $S R$ sustainedrelease formulation of $\beta$-alanine, $R R$ rapid-release formulation of $\beta$-alanine, $P L A$ placebo

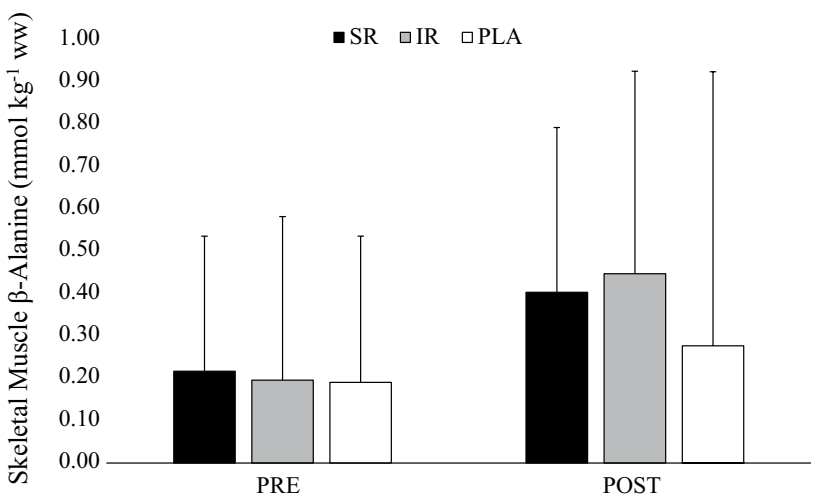

Fig. 4 Unadjusted values for PRE- and POST-supplementation skeletal muscle $\beta$-alanine content (adjusted PRE mean $=0.15 \mathrm{mmol} \mathrm{kg}^{-1}$ ww). Results are reported as mean \pm SD. PRE before 28 days of supplementation, POST after 28 days of supplementation, $S R$ sustainedrelease formulation of $\beta$-alanine, $R R$ rapid-release formulation of $\beta$-alanine, $P L A$ placebo 
partial $\left.\eta^{2}=0.007\right)$ between participants consuming the SR formulation $\left(0.28 \pm 0.12 \mathrm{mmol} \mathrm{kg}^{-1} \mathrm{ww}\right)$, RR formulation $\left(0.27 \pm 0.12 \mathrm{mmol} \mathrm{kg}^{-1} \mathrm{ww}\right)$ or PLA $\left(0.29 \pm 0.12 \mathrm{mmol} \mathrm{kg}^{-1} \mathrm{ww}\right)$ were noted at POST.

The average-adjusted muscle $\beta$-alanine content at PRE was $0.15 \pm 0.31 \mathrm{mmol} \cdot \mathrm{kg}^{-1}$ ww. No significant differences $\left(F=1.824, p=0.184\right.$, partial $\left.\eta^{2}=0.137\right)$ were noted between participants consuming the SR formulation $\left(0.42 \pm 0.36 \mathrm{mmol} \mathrm{kg}^{-1} \mathrm{ww}\right)$, the RR formulation $\left(0.50 \pm 0.37 \mathrm{mmol} \mathrm{kg} \mathrm{kg}^{-1} \mathrm{ww}\right)$ or PLA $\left(0.14 \pm 0.36 \mathrm{mmol} \mathrm{kg}^{-1} \mathrm{ww}\right)$ at POST

\section{Maximal voluntary isometric contraction peak torque}

Changes in $\triangle \mathrm{PT}$ are depicted in Fig. 5. At PRE, the average-adjusted $\triangle \mathrm{PT}$ following the muscle-damaging protocol was $-91.39 \pm 48.61 \mathrm{Nm}$. Comparison of groups at POST revealed significant differences $(F=6.145, p=0.007$, partial $\left.\eta^{2}=0.348\right)$ in $\Delta$ PT. Following the 28 -day supplementation period, $\triangle \mathrm{PT}$ was significantly less for both participants consuming the SR $(-64.74 \pm 27.68 \mathrm{Nm}, p=0.002$, $d=1.58)$ and RR formulations $(-74.61 \pm 29.94 \mathrm{Nm}$, $p=0.024, d=1.19)$ compared to PLA $(-110.05 \pm 29.58)$. No significant differences were noted in $\triangle \mathrm{PT}$ between participants consuming the SR and RR formulations $(p=0.452, d=0.34)$; however, this represented a smallmedium effect size.

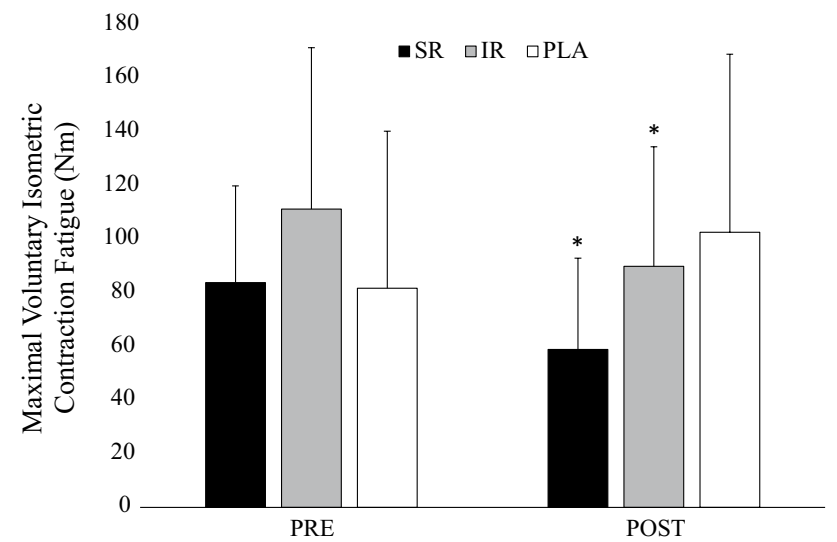

Fig. 5 Unadjusted values for PRE- and POST-supplementation decline in peak torque. *Significantly greater than placebo after adjusting for the PRE covariate (adjusted PRE mean=91.40 Nm). Results are reported as mean $\pm \mathrm{SD}$. PRE before 28 days of supplementation, POST after 28 days of supplementation, $S R$ sustainedrelease formulation of $\beta$-alanine, $R R$ rapid-release formulation of $\beta$-alanine, $P L A$ placebo

\section{Discussion}

Initial studies reporting significant increases in skeletal muscle carnosine from $\beta$-alanine supplementation used an RR formulation (Harris et al. 2006; Hill et al. 2007). Symptoms of paresthesia were a common side effect associated with $\beta$-alanine ingestion, with greater symptoms associated with larger daily doses (Decombaz et al. 2012; Harris et al. 2006; McCormack et al. 2013). More recently, an SR form of $\beta$-alanine has become available, which delays the release of $\beta$-alanine and prevents or attenuates symptoms of paresthesia (Decombaz et al. 2012; del Favero et al. 2012; Hoffman et al. 2014, 2015a). The present investigation is an initial foray into exploring whether differences in $\beta$-alanine formulation differ with regard to their effectiveness in increasing skeletal muscle carnosine and subsequent performance improvement. The main findings of this study indicate that daily ingestion of $6 \mathrm{~g}$ SR formulation of $\beta$-alanine for a period of 28 days significantly increased skeletal muscle carnosine content and attenuated the decline in PT after a unilateral, lower body, muscle-fatiguing protocol. Participants consuming the RR formulation experienced no statistically significant increases in muscle carnosine content, but had significantly attenuated changes in PT. Although changes in muscle carnosine and performance results were not significantly different between the different $\beta$-alanine formulations, participants consuming the RR formulation reported significantly more side effects (e.g., symptoms of paresthesia) associated with consuming the supplement compared to participants consuming the SR formulation or PLA.

Elevations in muscle carnosine observed in this investigation were consistent with previous investigations (Harris et al. 2006; del Favero et al. 2012; Derave et al. 2007; Hill et al. 2007; Hoffman et al. 2015a). Participants supplementing with the SR formulation experienced an increase in unadjusted muscle carnosine content of $50.1 \%$ (3.87 $\mathrm{mmol} \mathrm{kg}^{-1} \mathrm{ww}$ ), while those participants supplementing with the RR formulation increased muscle carnosine content by $37.9 \%\left(2.62 \mathrm{mmol} \mathrm{kg}^{-1} \mathrm{ww}\right)$, with no significant difference observed between the groups. Previous studies using dosing strategies ranging from 4.0-6.4 $\mathrm{g} \mathrm{day}^{-1}$ for 4-10 weeks have reported increases in muscle carnosine (within the vastus lateralis) ranging from 6.55-17.0 mmol kg-1 $\mathrm{dw}$ (Hoffman et al. 2018), equivalent to a $1.52-3.95 \mathrm{mmol} \mathrm{kg}^{-1} \mathrm{ww}$ increase. Interestingly, only participants consuming the SR formulation had elevations in muscle carnosine content that were significantly different than those supplementing with the PLA, while no significant differences were noted between those supplementing with the RR formulation and those participants 
supplementing with the PLA. However, a large effect size was observed for changes in carnosine content in participants consuming the RR formulation compared to PLA, suggesting that carnosine content was elevated with the RR formulation. Additionally, despite no significant differences in muscle carnosine at POST were noted between participants consuming the SR and RR formulations, a $16.4 \%$ difference in the change in muscle carnosine between the groups does provide an interesting outcome. The estimated retention of $\beta$-alanine was calculated at $6.8 \pm 8.2$ and $5.5 \pm 6.1 \%$ for participants consuming SR and RR formulations, respectively. Although these differences were not significant ( $p=0.617)$, it is important to keep in mind that this study was not statistically powered to examine differences in retention time between the two formulations.

Previous research comparing the effects of the SR and RR formulations ( $3.2 \mathrm{~g} \mathrm{day}^{-1}$ for 46 days) reported similar increases in carnosine content with both formulations (Stegen et al. 2013). However, that study utilized a tablet form for the SR delivery, while the RR formulation was powder provided in a gel capsule. Whether the delivery system had any influence is not well understood. However, the current study appears to support the work of Stegen at al. (2013). Although no significant differences were noted in the change in carnosine content in participants ingesting the SR and RR $\beta$-alanine formulations at POST, a small-medium effect size was observed for carnosine elevations between groups. Additionally, the significant increase in muscle carnosine in the SR group from PRE compared to the lack of any significant increase seen in the RR group suggests that the pharmacokinetics of $\beta$-alanine release from the SR formulation may be more effective for increasing muscle carnosine content. The RR formulation results in a shorter plasma $\beta$-alanine half-life but larger peak, while the area under the plasma concentration curve is the same as with the SR formulation (Decombaz et al. 2012). SR formulations may increase the time over which plasma $\beta$-alanine is elevated (Decombaz et al. 2012), possibly enhancing the potential for $\beta$-alanine to be transported into the muscle to form carnosine. With the RR formulation, a sharp increase in plasma free $\beta$-alanine may result in an increase in the binding of $\beta$-alanine to $\mathrm{MrgD}$ receptors, preventing $\beta$-alanine transport into the muscle and resulting in increased feelings of paresthesia (Bader et al. 2014; Crozier et al. 2007). The pharmokinetic difference between the RR and SR formulations may explain part of the mechanism behind the $16.4 \%$ difference noted between the two $\beta$-alanine groups in the response of carnosine synthesis.

The inability to achieve any statistically significant differences in carnosine elevations between participants consuming SR and RR formulations may also be a function of supplementation duration. While the absolute difference in the increase in muscle carnosine was small at 28 days, forward

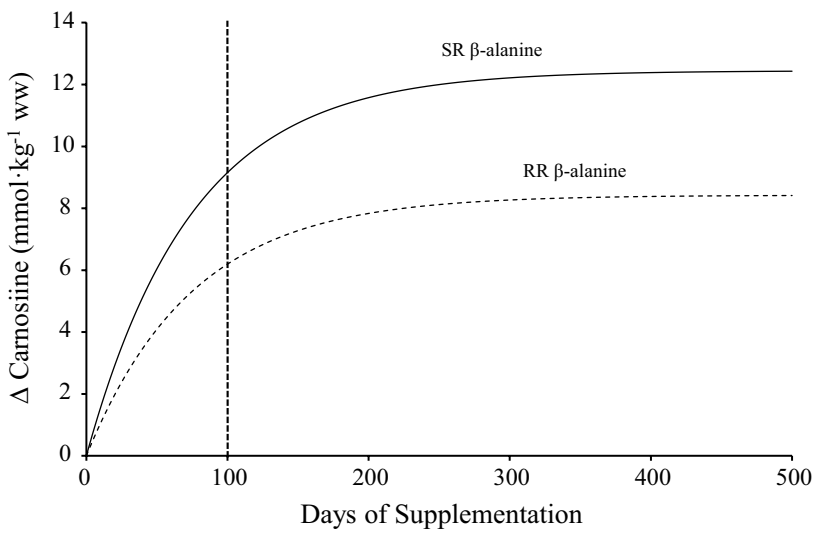

Fig. 6 Forward projection of changes in intramuscular carnosine concentrations using a rapid-release formulation (RR) compared to a sustained-release formulation (SR) over different durations of $\beta$-alanine supplementation based off of the kinetics presented by Spelnikov and Harris (2018)

projection of the change using the model of Spelnikov and Harris (2018), indicates a much greater difference appearing with longer duration of supplementation (see Fig. 6). Forward projection of the change has been calculated from the formula:

$\Delta$ Carnosine $=\left(k_{\mathrm{f}} / k_{\mathrm{d}}\right) \cdot\left(1-\exp \left(-k_{\mathrm{d}} \cdot t\right)\right)$,

where $k_{\mathrm{f}}$ is the zero-order rate constant for carnosine formation. $k_{\mathrm{d}}$ is the first-order rate constant for carnosine decay as $\mathrm{d}^{-1} . t$ is the amount of days of $\beta$-alanine supplementation.

From Spelnikov and Harris (2018), $k_{\mathrm{d}}$ is assumed to be 0.0133 , which corresponds to a post-supplementation halflife of 52 days. $k_{\mathrm{f}}$ has been calculated from Spelnikov and Harris (2018):

$k_{\mathrm{f}}=\left(k_{\mathrm{d}} \cdot \Delta\right.$ Carnosine $) /\left(1-\exp \left(-k_{\mathrm{d}} \cdot t\right)\right)$

Considering the change in carnosine at 28 days was 3.87 and $2.62 \mathrm{mmol} . \mathrm{kg}^{-1} \mathrm{ww}$ for participants consuming SR and $\mathrm{RR}$, respectively, the estimated $k_{\mathrm{f}}$ for SR and RR was calculated to be 0.16554 and 0.11207 , respectively. Figure 6 indicates that within 100 days of supplementation, the increase in muscle carnosine could be as high as $9.15 \mathrm{mmol} \mathrm{kg}^{-1}$ ww for SR compared to $6.20 \mathrm{mmol} \mathrm{kg}^{-1} \mathrm{ww}$ for RR. We acknowledge that this is quite speculative, especially in consideration that this is the first application of the Spelnikov and Harris model, uncertain whether the model would hold true for 100 days, and uncertain regarding the estimate of $k_{\mathrm{d}}$.

A recent investigation reported that 23 days of $\beta$-alanine supplementation $\left(6 \mathrm{~g} \mathrm{day}^{-1}\right)$ in its RR formulation may result in a significant decrease in muscle histidine content (Blancquaert et al. 2017). However, other investigations using the SR formulation reported no change in muscle histidine content (Church et al. 2017; Varanoske et al. 2017a). The results of the present investigation support the latter 
studies indicating that $\beta$-alanine supplementation does not reduce muscle histidine content, regardless of formulation. In the present study, greater levels of skeletal muscle histidine $\left(0.34 \pm 0.17 \mathrm{mmol} \mathrm{kg}^{-1} \mathrm{ww}\right)$ were noted at baseline compared to those of Blancquaert et al. (2017) $\left(0.20 \pm 0.06 \mathrm{mmol} \mathrm{kg}^{-1} \mathrm{ww}\right)$ and were similar to those previously reported in individuals with high muscle carnosine content (Varanoske et al. 2017a). Although speculative, it is possible that higher baseline levels of histidine, perhaps from dietary means, may provide for a sufficient reserve to prevent any significant declines when supplementing with $\beta$-alanine.

$\triangle \mathrm{PT}$ was significantly attenuated in participants consuming both the SR and RR formulations compared to PLA. Although no significant differences in $\triangle \mathrm{PT}$ were noted between the SR and RR formulations, an interesting finding was that the effect size seen in $\triangle \mathrm{PT}$ at POST between SR and RR was similar to the effect size determined for the differences in carnosine content at POST. This finding provides further support that increases in carnosine content may directly improve high-intensity exercise performance. Performance improvements observed in this investigation were consistent with other studies demonstrating the efficacy of $\beta$-alanine supplementation on attenuating fatigue during repeated maximal isokinetic contractions of the knee extensors (Derave et al. 2007; Varanoske et al. 2017a, b). However, the effect of $\beta$-alanine supplementation on isometric muscle performance is less clear. Derave et al. (2007) reported no differences in time to exhaustion during isometric endurance at $45 \%$ of maximal voluntary contraction of the knee extensors after $\beta$-alanine supplementation (4.8 $\mathrm{g} \mathrm{day}^{-1}$ for 4 weeks), whereas Sale et al. (2012) reported significant increases in time to exhaustion utilizing a similar exercise testing protocol after $\beta$-alanine supplementation ( $6.4 \mathrm{~g} \mathrm{day}^{-1}$ for 4 weeks). Sale et al. (2012) hypothesized that the discrepancy between the findings may be a result of the actual intensity of the exercise in the Derave et al. (2007) study, as it may have corresponded to a lower percentage of maximal voluntary contraction than intended. Ahlborg et al. (1972) predicted the time to exhaustion at $45 \%$ of maximal voluntary contraction to be about $80 \mathrm{~s}$, whereas the hold-time of participants in Derave et al. (2007) study lasted longer than $175 \mathrm{~s}$. Exercise of longer duration utilizes the oxidative system for metabolic energy production to a greater extent than shorter-duration exercise, leading to lower $\mathrm{H}^{+}$accumulation. Furthermore, our results contradict with those of Kendrick et al. (2008) who reported no improvement in quadriceps isokinetic force after $\beta$-alanine supplementation ( $6.4 \mathrm{~g} \mathrm{day}^{-1}$ for 10 weeks), and Hannah et al. (2015) and Jones et al. (2017), who reported no change in quadriceps maximal voluntary isometric force production or the force-frequency relationship after $\beta$-alanine supplementation ( $6.4 \mathrm{~g} \mathrm{day}^{-1}$ for 28 days). The differences in findings may be related to the exercises utilized, as the isometric protocols by Kendrick et al. (2008), Derave et al. (2007), Hannah et al. (2015) and Jones et al. (2017) may not have elicited the extent of fatigue to maximize the benefits achieved from a greater buffering capacity. Carnosine is known to enhance intracellular buffering of $\mathrm{H}^{+}$(Abe 2000), improving performance particularly during high-intensity anaerobic exercise, and $\beta$-alanine supplementation does not appear to have an effect on maximal strength production (Hoffman et al. 2008; Kendrick et al. 2008). Isometric exercise protocols consisting of only maximal contractions likely result in minimal changes in $\mathrm{H}^{+}$production.

One potential limitation of this investigation is that the validity of this double-blind experiment may have been compromised if participants knew which group they were in. Considering that all the participants in the RR group reported symptoms of paresthesia on at least half of the days that they supplemented, participants may not have been truly blinded to which group they were in, potentially influencing performance outcomes. Additionally, participants were not familiarized with the muscle-damaging protocol prior to testing, which may have affected the results. Furthermore, the unequal amount of subjects in each group and variability in the data, and small sample size may indicate that the study was underpowered to produce sufficient results.

In conclusion, this investigation demonstrated that supplementation of $6 \mathrm{~g} \mathrm{day}^{-1}$ of an SR formulation of $\beta$-alanine for a period of 28 days significantly increased skeletal muscle carnosine content and maximal isometric exercise performance after a muscle-fatiguing protocol. Although these results were not statistically different from individuals consuming an RR formulation of $\beta$-alanine, the $16.4 \%$ greater increase in muscle carnosine between participants consuming the SR and RR formulations may have practical significance for elite athletic populations in which small changes may have important performance effects. Future research needs to compare these different formulations on a competitive athletic population to further explore this potential advantage. Regardless, participants consuming the SR formulation experienced significantly less paresthesia compared to those consuming the RR form, making the SR form more practical and attractive for future use.

Acknowledgement The authors would like to thank Natural Alternatives International (Carlsbad, CA, USA; Grant Number 14248018) for providing support for this study. The funding source had no such involvement in collection, interpretation, or analysis of data, or of writing the report.

Author contribution ANV, JRH, DDC, RW, DHF and JRS conceived and designed the experiments; ANV, DDC, NAC, KMB, SJD, LPO, and VLD performed the experiments; ANV, JRH, and JRS analyzed the data; ANV. RCH and JRH wrote the paper. All authors approved the final version of the manuscript. 


\section{Compliance with ethical standards}

Conflict of interest RC.H. is a paid consultant to Natural Alternatives International. All other authors declare that they have no conflict of interest to report.

Ethical approval All procedures performed involved human participants and were in accordance with the ethical standards of the institutional and with the 1964 Helsinki Declaration and its later amendments or comparable ethical standards.

Informed consent Informed consent was obtained from all individual participants included in the study.

Open Access This article is distributed under the terms of the Creative Commons Attribution 4.0 International License (http://creativeco mmons.org/licenses/by/4.0/), which permits unrestricted use, distribution, and reproduction in any medium, provided you give appropriate credit to the original author(s) and the source, provide a link to the Creative Commons license, and indicate if changes were made.

\section{References}

Abe H (2000) Role of histidine-related compounds as intracellular proton buffering constituents in vertebrate muscle. Biochemistry 65:757-765

Ahlborg B, Bergstrom J, Hultman E, Nordesjo LO, Ekelund LG, Harris RC, Guarnieri G (1972) Muscle metabolism during isometric exercise performed at constant force. J Appl Physiol 33:224

Bader M, Alenina N, Andrade-Navarro MA, Santos RA (2014) MAS and its related $\mathrm{G}$ protein-coupled receptors, Mrgprs. Pharmacol Rev 66:1080-1105. https://doi.org/10.1124/pr.113.008136

Baguet A, Bourgois J, Vanhee L, Achten E, Derave W (2010) Important role of muscle carnosine in rowing performance. J Appl Physiol 109:1096-1101. https://doi.org/10.1152/japplphysiol.00141.2010

Barbato G, Barini EM, Genta G, Levi R (2011) Features and performance of some outlier detection methods. J Appl Stat 38:21332149. https://doi.org/10.1080/02664763.2010.545119

Beyer KS, Fukuda DH, Boone CH, Wells AJ, Townsend JR, Jajtner AR, Gonzalez AM, Fragala MS, Hoffman JR, Stout JR (2016) Short-term unilateral resistance training results in cross education of strength without changes in muscle size, activation, or endocrine response. J Strength Cond Res 30:1213-1223. https:// doi.org/10.1519/JSC.0000000000001219

Blancquaert L, Everaert I, Missinne M, Baguet A, Stegen S, Volkaert A, Petrovic M, Vervaet C, Achten E, De Maeyer M, De Henauw S, Derave W (2017) Effects of histidine and beta-alanine supplementation on human muscle carnosine storage. Med Sci Sports Exerc. https://doi.org/10.1249/MSS.0000000000001213

Church DD, Hoffman JR, Varanoske AN, Wang R, Baker KM, La Monica MB, Beyer KS, Dodd SJ, Oliveira LP, Harris RC, Fukuda DH, Stout JR (2017) Comparison of two beta-alanine dosing protocols on muscle carnosine elevations. J Am Coll Nutr 36:608-616. https ://doi.org/10.1080/07315724.2017.1335250

Cohen J (1969) Statistical Power Analysis for the Behavioral Sciences. Academic Press, New York

Crozier RA, Ajit SK, Kaftan EJ, Pausch MH (2007) MrgD activation inhibits KCNQ/M-currents and contributes to enhanced neuronal excitability. J Neurosci 27:4492-4496. https://doi.org/10.1523/ JNEUROSCI.4932-06.2007

de Salles Painelli V, Roschel H, Jesus F, Sale C, Harris RC, Solis MY, Benatti FB, Gualano B, Lancha AH Jr, Artioli GG (2013) The ergogenic effect of beta-alanine combined with sodium bicarbonate on high-intensity swimming performance. Appl Physiol Nutr Metab Physiologie appliquee, nutrition et metabolisme 38:525532. https://doi.org/10.1139/apnm-2012-0286

Decombaz J, Beaumont M, Vuichoud J, Bouisset F, Stellingwerff T (2012) Effect of slow-release beta-alanine tablets on absorption kinetics and paresthesia. Amino Acids 43:67-76. https://doi. org/10.1007/s00726-011-1169-7

del Favero S, Roschel H, Solis MY, Hayashi AP, Artioli GG, Otaduy MC, Benatti FB, Harris RC, Wise JA, Leite CC, Pereira RM, de Sa-Pinto AL, Lancha-Junior AH, Gualano B (2012) Betaalanine (Carnosyn) supplementation in elderly subjects (6080 years): effects on muscle carnosine content and physical capacity. Amino Acids 43:49-56. https://doi.org/10.1007/s0072 6-011-1190-x

Derave W, Ozdemir MS, Harris RC, Pottier A, Reyngoudt H, Koppo K, Wise JA, Achten E (2007) beta-Alanine supplementation augments muscle carnosine content and attenuates fatigue during repeated isokinetic contraction bouts in trained sprinters. $\mathrm{J}$ Appl Physiol 103:1736-1743. https://doi.org/10.1152/japplphysi ol.00397.2007

Dunnett M, Harris RC (1999) Influence of oral beta-alanine and L-histidine supplementation on the carnosine content of the gluteus medius. Equine Vet J Suppl 30:499-504

Hannah R, Stannard RL, Minshull C, Artioli GG, Harris RC, Sale C (2015) $\beta$-Alanine supplementation enhances human skeletal muscle relaxation speed but not force production capacity. J Appl Physiol 118:604-612. https://doi.org/10.1152/japplphysiol.00991 .2014

Harris RC, Tallon MJ, Dunnett M, Boobis L, Coakley J, Kim HJ, Fallowfield JL, Hill CA, Sale C, Wise JA (2006) The absorption of orally supplied beta-alanine and its effect on muscle carnosine synthesis in human vastus lateralis. Amino Acids 30:279-289. https://doi.org/10.1007/s00726-006-0299-9

Hill CA, Harris RC, Kim HJ, Harris BD, Sale C, Boobis LH, Kim CK, Wise JA (2007) Influence of beta-alanine supplementation on skeletal muscle carnosine concentrations and high intensity cycling capacity. Amino Acids 32:225-233. https://doi. org/10.1007/s00726-006-0364-4

Hoffman JR, Ratamess NA, Faigenbaum AD, Ross R, Kang J, Stout JR, Wise JA (2008) Short-duration beta-alanine supplementation increases training volume and reduces subjective feelings of fatigue in college football players. Nutr Res 28:31-35. https://doi. org/10.1016/j.nutres.2007.11.004

Hoffman JR, Landau G, Stout JR, Dabora M, Moran DS, Sharvit N, Hoffman MW, Ben Moshe Y, McCormack WP, Hirschhorn G, Ostfeld I (2014) $\beta$-alanine supplementation improves tactical performance but not cognitive function in combat soldiers. J Int Soc Sports Nutr 11:15. https://doi.org/10.1186/1550-2783-11-15

Hoffman JR, Landau G, Stout JR, Hoffman MW, Shavit N, Rosen P, Moran DS, Fukuda DH, Shelef I, Carmom E, Ostfeld I (2015a) $\beta$-Alanine ingestion increases muscle carnosine content and combat specific performance in soldiers. Amino Acids 47:627-636. https://doi.org/10.1007/s00726-014-1896-7

Hoffman JR, Stout JR, Harris RC, Moran DS (2015b) $\beta$-Alanine supplementation and military performance. Amino Acids 47:24632474. https://doi.org/10.1007/s00726-015-2051-9

Hoffman JR, Varanoske A, Stout JR (2018) Effects of beta-alanine supplementation on carnosine elevation and physiological performance. Adv Food Nutr Res 84:183-206. https://doi.org/10.1016/ bs.afnr.2017.12.003

Huck SW, McLean RA (1975) Using a repeated measures ANOVA to analyze data from a pretest-posttest design-potentially confusing task. Psychol Bull 82:511-518. https://doi.org/10.1037/H0076767

Jones RL, Barnett CT, Davidson J, Maritza B, Fraser WD, Harris R, Sale C (2017) beta-alanine supplementation improves in vivo 
fresh and fatigued skeletal muscle relaxation speed. Eur J Appl Physiol 117:867-879. https://doi.org/10.1007/s00421-017-3569-1

Kendrick IP, Harris RC, Kim HJ, Kim CK, Dang VH, Lam TQ, Bui TT, Smith M, Wise JA (2008) The effects of 10 weeks of resistance training combined with beta-alanine supplementation on whole body strength, force production, muscular endurance and body composition. Amino Acids 34:547-554. https://doi.org/10.1007/ s00726-007-0008-3

Kriengsinyos W, Rafii M, Wykes LJ, Ball RO, Pencharz PB (2002) Long-term effects of histidine depletion on whole-body protein metabolism in healthy adults. J Nutr 132:3340-3348

McCormack WP, Stout JR, Emerson NS, Scanlon TC, Warren AM, Wells AJ, Gonzalez AM, Mangine GT, Robinson EHt, Fragala MS, Hoffman JR (2013) Oral nutritional supplement fortified with beta-alanine improves physical working capacity in older adults: a randomized, placebo-controlled study. Exp Gerontol 48:933-939. https://doi.org/10.1016/j.exger.2013.06.003

Mora L, Sentandreu MA, Toldra F (2007) Hydrophilic chromatographic determination of carnosine, anserine, balenine, creatine, and creatinine. J Agric Food Chem 55:4664-4669. https://doi. org/10.1021/jf0703809

Sale C, Hill CA, Ponte J, Harris RC (2012) $\beta$-alanine supplementation improves isometric endurance of the knee extensor muscles. J Int Soc Sports Nutr 9(1):26. https://doi.org/10.1186/1550-2783-9-26

Saunders B, Elliott-Sale K, Artioli GG, Swinton PA, Dolan E, Roschel H, Sale C, Gualano B (2017) beta-alanine supplementation to improve exercise capacity and performance: a systematic review and meta-analysis. Br J Sports Med 51:658. https://doi. org/10.1136/bjsports-2016-096396

Smith-Ryan AE, Fukuda DH, Stout JR, Kendall KL (2012) Highvelocity intermittent running: effects of beta-alanine supplementation. J Strength Cond Res 26:2798-2805. https://doi.org/10.1519/ JSC.0b013e 318267922b

Spelnikov D, Harris RC (2018) A kinetic model of carnosine synthesis in human skeletal muscle. Amino Acids. In Press
Stegen S, Blancquaert L, Everaert I, Bex T, Taes Y, Calders P, Achten E, Derave W (2013) Meal and beta-alanine coingestion enhances muscle carnosine loading. Med Sci Sports Exerc 45:1478-1485. https://doi.org/10.1249/MSS.0b013e31828ab073

Stout JR, Cramer JT, Zoeller RF, Torok D, Costa P, Hoffman JR, Harris RC, O'Kroy J (2007) Effects of beta-alanine supplementation on the onset of neuromuscular fatigue and ventilatory threshold in women. Amino Acids 32:381-386. https://doi.org/10.1007/s0072 6-006-0474-z

Stout JR, Graves BS, Smith AE, Hartman MJ, Cramer JT, Beck TW, Harris RC (2008) The effect of beta-alanine supplementation on neuromuscular fatigue in elderly (55-92 years): a doubleblind randomized study. J Int Soc Sports Nutr 5:21. https://doi. org/10.1186/1550-2783-5-21

Townsend JR, Hoffman JR, Fragala MS, Oliveira LP, Jajtner AR, Fukuda DH, Stout JR (2016) A microbiopsy method for immunohistological and morphological analysis: a pilot study. Med Sci Sports Exerc 48:331-335. https://doi.org/10.1249/MSS.00000 00000000772

Varanoske AN, Hoffman JR, Church DD, Coker NA, Baker KM, Dodd SJ, Oliveira LP, Dawson VL, Wang R, Fukuda DH, Stout JR (2017a) Beta-Alanine supplementation elevates intramuscular carnosine content and attenuates fatigue in men and women similarly but does not change muscle L-histidine content. Nutr Res 48:16-25. https://doi.org/10.1016/j.nutres.2017.10.002

Varanoske AN, Hoffman JR, Church DD, Wang R, Baker KM, Dodd SJ, Coker NA, Oliveira LP, Dawson VL, Fukuda DH, Stout JR (2017b) Influence of skeletal muscle carnosine content on fatigue during repeated resistance exercise in recreationally active women. Nutrients. https://doi.org/10.3390/Nu9090988

Vincent WJ, Weir JP (2012) Statistics in kinesiology. Human Kinetics, Champaign 